\title{
2D Four-Channel Perfect Reconstruction Filter Bank Realized with the 2D Lattice Filter Structure
}

\author{
S. Sezen and A. Ertüzün \\ Department of Electrical and Electronics Engineering, Boğaziçi University, Bebek 34342, Istanbul, Turkey
}

Received 15 April 2005; Revised 14 December 2005; Accepted 13 January 2006

Recommended for Publication by Stephen Marshall

A novel orthogonal 2D lattice structure is incorporated into the design of a nonseparable 2D four-channel perfect reconstruction filter bank. The proposed filter bank is obtained by using the polyphase decomposition technique which requires the design of an orthogonal 2D lattice filter. Due to constraint of perfect reconstruction, each stage of this lattice filter bank is simply parameterized by two coefficients. The perfect reconstruction property is satisfied regardless of the actual values of these parameters and of the number of the lattice stages. It is also shown that a separable 2D four-channel perfect reconstruction lattice filter bank can be constructed from the 1D lattice filter and that this is a special case of the proposed 2D lattice filter bank under certain conditions. The perfect reconstruction property of the proposed 2D lattice filter approach is verified by computer simulations.

Copyright @ 2006 Hindawi Publishing Corporation. All rights reserved.

\section{INTRODUCTION}

Subband decomposition and coding of images have become quite popular in the last two decades. Subband coding has first been applied to speech signals by Crochiere et al. [1] and has later gained attention as a powerful method for compression of still images and video [2-4]. While most of the research in the area of subband decomposition concentrated on 1D signals and on separable approaches for multidimensional signals in the eighties, the nonseparable approaches have prevailed in the area of $2 \mathrm{D}$ filter banks from nineties onward. Daubechies [5] and Mallat [6] have developed the theory of wavelets and have shown that subband coding and wavelets are closely related.

In subband signal coding, the basic objective is to concentrate the signal energy in as few subspectra or subbands as possible for efficient transmission of information. The uneven distribution of signal energy over the frequency band provides the basis for source compression techniques, thus data compression is the driving motivation for subband signal coding. In subband coding, the frequency band of the signal is first divided into a set of uncorrelated frequency bands by filtering and then each of these subbands is encoded using a bit allocation rationale matched to the signal energy in that subband.

Multirate filter banks find applications in subband decomposition systems. The complete filter bank is composed of two sections: the analysis section which decomposes the signal into a set of subband components and the synthesis section which reconstructs the signal from its components. The subband analysis and synthesis filters should be designed to be alias-free and should satisfy the perfect signal reconstruction property. The simultaneous cancellation of aliasing as well as amplitude and phase distortions leads to perfect reconstruction (PR) filter banks which are suitable for hierarchical subband coding and multiresolution signal decomposition.

In a 1D two-channel filter bank decomposition system, the input signal can be split into lowpass and highpass subbands using quadrature mirror filters (QMF). Design of quadrature mirror filter banks (QMFB) in the frequency domain and in the time domain has been presented in $[7,8]$, respectively. The PR property and the requirements for $1 \mathrm{D}$ two-channel finite impulse response QMFB have been first obtained by Smith and Barnwell [9] and then have been thoroughly treated by Vaidyanathan [10]. The results of 1D two-band filter banks are extended to $M$-band without using separable filters and more general PR conditions are obtained in $[11,12]$. Nguyen and Vaidyanathan [13] have relaxed the power complementary requirements and the QMF restrictions of the filters and have obtained two-channel PR structures which yield linear phase analysis and synthesis filters for an arbitrary number of channels. 
Vaidyanathan and Hoang [14] have used a 1D lattice filter structure for the design of two-channel QMFBs, which satisfied a sufficient condition for PR. The lattice structure has a hierarchical property so that a higher-order PR QMFB can be constructed from a lower-order PR QMFB simply by adding more lattice sections. Vaidyanathan later extended this structure to $M$-channel using an optimization technique with $M \times M$ orthogonal matrix as presented in [15].

Smith and Eddins [4], Tay and Kingsbury [16] have developed subband filters which have PR properties by using different structures. Simoncelli [17], on the other hand, has developed a nonseparable multidimensional filter bank, which does not satisfy the conditions for PR. Bamberger and Smith [18] have introduced fan filter banks with wedgeshaped subbands. Other techniques have been proposed for the design of diamond filters, fan filters, and directional filters that originate from a 1D design [19-22]. Many researchers have investigated the theory, the design methods, and the structures for linear phase PR filter banks [23-39] and solutions have been found.

For 2D subband decomposition systems, there exist two different approaches: separable and nonseparable. Most of the early reported work on subband coding of images and image sequences are based on separable filtering using 1D QMFBs. However, it is only proper that 2D signals, such as images, should be processed with truly $2 \mathrm{D}$ systems. An independent extension of QMFBs to the multi-dimensional case has been published by Vetterli [2] who has claimed that separable filters are necessary and sufficient for the most natural four-band extension of the standard two-band QMFBs. Woods and O'neil [3] have derived conditions for nonseparable filters and proved that separability is not necessary for the design of 2D filter banks. Their approach, however, has not supported PR in the filter banks.

Motivated by the success of Vaidyanathan and Hoang [14] who have used 1D lattice filter structures for the design of 1D two-channel PR QMFBs, we examine the use of 2D lattice filter structure for the design of $2 \mathrm{D}$ four-channel PR filter banks. To the best of the authors' knowledge, 2D lattice filter structures have not been used in the context of PR filter banks. As they are 2D structures, it is extremely important to use $2 \mathrm{D}$ lattice filters to fully exploit the properties of $2 \mathrm{D}$ signals.

The theory of 2D lattice filters is developed as a natural extension of the $1 \mathrm{D}$ lattice filter theory. $1 \mathrm{D}$ and $2 \mathrm{D}$ lattice filters have similar properties such as the hierarchical property, in the sense that higher-order filters can be obtained from lower-order filters simply by concatenating new lattice stages to the existing ones. The utility of a $2 \mathrm{D}$ lattice filter in the context of subband decomposition is that the resulting filter structure is nonseparable. The $2 \mathrm{D}$ threeparameter lattice filter (3PLF) structure developed by Parker and Kayran [40] generates four 2D prediction error filters (quadrant filters) simultaneously and combines them into a single structure in the form of a lattice filter, therefore, the realization of four-channel filter bank by use of the 3PLF is very natural. However, the prediction filters are not orthogonal and the PR property is not satisfied. Thus, based on the concepts of structural stability given in [41], we design four orthogonal prediction filters and combine them to get a modified 3PLF structure-an orthogonal 2D lattice filter. We then use this orthogonal 2D lattice filter structure for the design of a $2 \mathrm{D}$ four-channel nonseparable PR lattice (NSPRL) filter bank [42]. As a consequence of the PR property, the proposed NSPRL structure has two independent parameters rather than three at each stage.

In this paper, we also develop a 2D four-channel separable perfect reconstruction lattice (SPRL) filter structure using the $1 \mathrm{D}$ lattice filter design given in [14]. We then show that the SPRL filter is a special case of the proposed NSPRL filter under certain conditions.

This paper is organized as follows. In Section 2, the background material on the 3PLF is given. In Section 3, the proposed 2D four-channel NSPRL filter bank is developed; the constraints required in order to satisfy the PR property and the computation of the lattice filter coefficients are presented. The relationship between the SPRL and the NSPRL filter banks is also given in this section. In Section 4, the results of the computer simulations are given and the PR property of the proposed structure is verified. Finally, the conclusions are drawn in Section 5.

\section{BACKGROUND MATERIAL ON THREE-PARAMETER LATTICE FILTER STRUCTURE}

Parker and Kayran [40] have introduced the concept of four prediction error fields which are combined into a quarterplane 2D lattice filter structure. This filter has three reflection coefficients at each stage and it is developed assuming that the input data has four-quadrant symmetry. Thus

$$
r\left(l_{1}, l_{2}\right)=r\left(l_{1},-l_{2}\right)=r\left(-l_{1}, l_{2}\right)=r\left(-l_{1},-l_{2}\right),
$$

where $r(\cdot, \cdot)$ 's are the correlations of the 2D data for appropriate lags. Due to the four-quadrant symmetry, the 3PLF is restricted to have one set of reflection coefficients at each lattice stage.

The 3PLF structure [40] which generates four prediction error fields can be represented by the following recursive input/output equation:

$$
\begin{aligned}
{\left[\begin{array}{c}
e_{00}^{(m+1)}\left(n_{1}, n_{2}\right) \\
e_{10}^{(m+1)}\left(n_{1}, n_{2}\right) \\
e_{11}^{(m+1)}\left(n_{1}, n_{2}\right) \\
e_{01}^{(m+1)}\left(n_{1}, n_{2}\right)
\end{array}\right]=} & {\left[\begin{array}{cccc}
1 & -k_{1}^{(m+1)} & -k_{2}^{(m+1)} & -k_{3}^{(m+1)} \\
-k_{1}^{(m+1)} & 1 & -k_{3}^{(m+1)} & -k_{2}^{(m+1)} \\
-k_{2}^{(m+1)} & -k_{3}^{(m+1)} & 1 & -k_{1}^{(m+1)} \\
-k_{3}^{(m+1)} & -k_{2}^{(m+1)} & -k_{1}^{(m+1)} & 1
\end{array}\right] } \\
& \times\left[\begin{array}{c}
e_{00}^{(m)}\left(n_{1}, n_{2}\right) \\
e_{10}^{(m)}\left(n_{1}-1, n_{2}\right) \\
e_{11}^{(m)}\left(n_{1}-1, n_{2}-1\right) \\
e_{01}^{(m)}\left(n_{1}, n_{2}-1\right)
\end{array}\right],
\end{aligned}
$$


where $k_{j}^{(m+1)}$, s are the lattice reflection coefficients of the $(m+$ 1)th stage where $(m)=\left(m_{1}, m_{2}\right)$ with $(m+1)=\left(m_{1}+1, m_{2}+\right.$ 1) and $n_{1}=1, \ldots, N_{1}, n_{2}=1, \ldots, N_{2}, m=1, \ldots, M-1$, and $M$ is the length of the filter. The initialization is as follows:

$$
\begin{aligned}
e_{00}^{(0)}\left(n_{1}, n_{2}\right) & =e_{10}^{(0)}\left(n_{1}, n_{2}\right)=e_{11}^{(0)}\left(n_{1}, n_{2}\right) \\
& =e_{01}^{(0)}\left(n_{2}, n_{2}\right)=x\left(n_{1}, n_{2}\right) .
\end{aligned}
$$

The error fields $e_{00}^{(m)}\left(n_{1}, n_{2}\right), e_{10}^{(m)}\left(n_{1}, n_{2}\right), e_{11}^{(m)}\left(n_{1}, n_{2}\right)$, and $e_{01}^{(m)}\left(n_{1}, n_{2}\right)$ correspond to the first, the second, the third, and the fourth quarter plane prediction error fields at the output of the $m$ th lattice stage, respectively, and $x\left(n_{1}, n_{2}\right)$ represents the $2 \mathrm{D}$ input data.

At each stage, the mean square value of the prediction error fields is minimized with respect to the reflection coefficients and the following normal equations are obtained:

$$
\mathbf{R}^{(m)} \mathbf{k}^{(m+1)}=\boldsymbol{\rho}^{(m)} .
$$

Here, $\mathbf{R}^{(m)}$ and $\boldsymbol{\rho}^{(m)}$ are, the symmetric autocorrelation matrix and the cross-correlation vector of prediction error fields of stage $(m)$, respectively, while $\mathbf{k}^{(m+1)}$ is the reflection coefficient vector of stage $(m+1)$, given as

$$
\mathbf{k}^{(m+1)}=\left[\begin{array}{lll}
k_{1}^{(m+1)} & k_{2}^{(m+1)} & k_{3}^{(m+1)}
\end{array}\right]^{T} .
$$

The correlations between the prediction error fields, $\phi_{e_{i j} e_{p q}}^{(m)}$ 's, are given as

$$
\begin{gathered}
\phi_{e_{i j} e_{p q}}^{(m)}=E\left[e_{i j}^{(m)}\left(n_{1}-i, n_{2}-j\right) e_{p q}^{(m)}\left(n_{1}-p, n_{2}-q\right)\right] \\
(i, j, p, q) \in(0,1) .
\end{gathered}
$$

The 2D transfer functions for the quadrant filters can be determined in terms of the lattice coefficients of the relevant stage. The $\left(m_{l}+1\right)$ th stage coefficient matrix for the forward $2 \mathrm{D}$ transfer function is given by

$\mathbf{B}_{00}^{(m+1)}=\widehat{\mathbf{B}}_{00}^{(m+1)}-k_{1}^{(m+1)} \widehat{\mathbf{B}}_{10}^{(m+1)}-k_{2}^{(m+1)} \widehat{\mathbf{B}}_{11}^{(m+1)}-k_{3}^{(m+1)} \widehat{\mathbf{B}}_{01}^{(m+1)}$,

where the augmented matrix for the forward prediction error filter is defined as follows:

$$
\widehat{\mathbf{B}}_{00}^{(m+1)}=\left[\begin{array}{cc}
\mathbf{B}_{00}^{(m)} & \mathbf{0} \\
\mathbf{0}^{T} & 0
\end{array}\right] .
$$

The coefficient matrix of the first stage of the forward prediction error filter is defined as

$$
\mathbf{B}_{00}^{(1)} \equiv\left[\begin{array}{cc}
1 & -k_{01}^{(1)} \\
-k_{10}^{(1)} & -k_{11}^{(1)}
\end{array}\right] .
$$

The coefficient matrices related to the backward prediction error filters, namely, $\mathbf{B}_{10}^{(1)}, \mathbf{B}_{11}^{(1)}$, and $\mathbf{B}_{01}^{(1)}$ are row, row and column, and column reversals of the matrix in (9), respectively. The augmented matrices $\widehat{\mathbf{B}}_{10}^{(m+1)}, \widehat{\mathbf{B}}_{11}^{(m+1)}$, and $\widehat{\mathbf{B}}_{01}^{(m+1)}$ are related to $\widehat{\mathbf{B}}_{00}^{(m+1)}$ by similar row and column operations [40]. The transfer functions of the forward and the backward prediction error filters can be defined as follows:

$$
\begin{array}{r}
H_{00}^{(m+1)}\left(z_{1}, z_{2}\right)=\sum_{j=0}^{m+1} \sum_{l=0}^{m+1} b_{00}^{(m+1)}(j, l) z_{1}^{-j} z_{2}^{-l}, \\
H_{10}^{(m+1)}\left(z_{1}, z_{2}\right)=z_{1}^{-(m+1)} H_{00}^{(m+1)}\left(z_{1}^{-1}, z_{2}\right), \\
H_{11}^{(m+1)}\left(z_{1}, z_{2}\right)=z_{1}^{-(m+1)} z_{2}^{-(m+1)} H_{00}^{(m+1)}\left(z_{1}^{-1}, z_{2}^{-1}\right), \\
H_{01}^{(m+1)}\left(z_{1}, z_{2}\right)=z_{2}^{-(m+1)} H_{00}^{(m+1)}\left(z_{1}, z_{2}^{-1}\right),
\end{array}
$$

where $b_{00}^{(m+1)}(j, l)$ 's are the coefficients of the $(m+1)$ th order lattice filter that can be obtained from the matrix defined in (7).

The filters, whose coefficient matrices are defined by (9) or by row, row and column, and column reversals of it are not orthogonal as defined by Lev-Ari and Parker [41]. In [43], it is shown that many different 3PLFs can be generated which satisfy the constraint of orthogonality and three such structures are given. Motivated by this, in the subsequent sections, first an orthogonal 2D lattice filter structure is developed and then it used in the 2D four-channel NSPRL filter bank.

\section{DESIGN OF THE PROPOSED 2D FOUR-CHANNEL NSPRL FILTER BANK}

\subsection{Orthogonal 2D lattice filter structure}

In this section, we derive the $2 \mathrm{D}$ orthogonal lattice filter structure by modifying the 3PLF using the polyphase decomposition technique.

The conventional four-channel filter bank is designed as shown in Figure 1 where $H_{0}\left(z_{1}, z_{2}\right), H_{1}\left(z_{1}, z_{2}\right), H_{2}\left(z_{1}, z_{2}\right)$, and $H_{3}\left(z_{1}, z_{2}\right)$ are the four analysis filters that split the spectrum into four subbands. The input signal is filtered with the subband filters and then downsampled 2-by-2 in the horizontal and vertical directions, respectively, and the output signals of the analysis stage, namely, $p_{0}\left(n_{1}, n_{2}\right), p_{1}\left(n_{1}, n_{2}\right)$, $p_{2}\left(n_{1}, n_{2}\right)$, and $p_{3}\left(n_{1}, n_{2}\right)$ are generated. In the synthesis section, these signals are first upsampled, then processed by the interpolation filters and finally summed to yield the reconstructed signal $\widehat{x}\left(n_{1}, n_{2}\right)$.

Our aim in introducing the $2 \mathrm{D}$ lattice filter approach into the 2D four-channel PR filter bank is to combine the four analysis subband filters into a single structure in the lattice form and to obtain high-order filter banks from the loworder filter banks using the hierarchical property of the lattice 


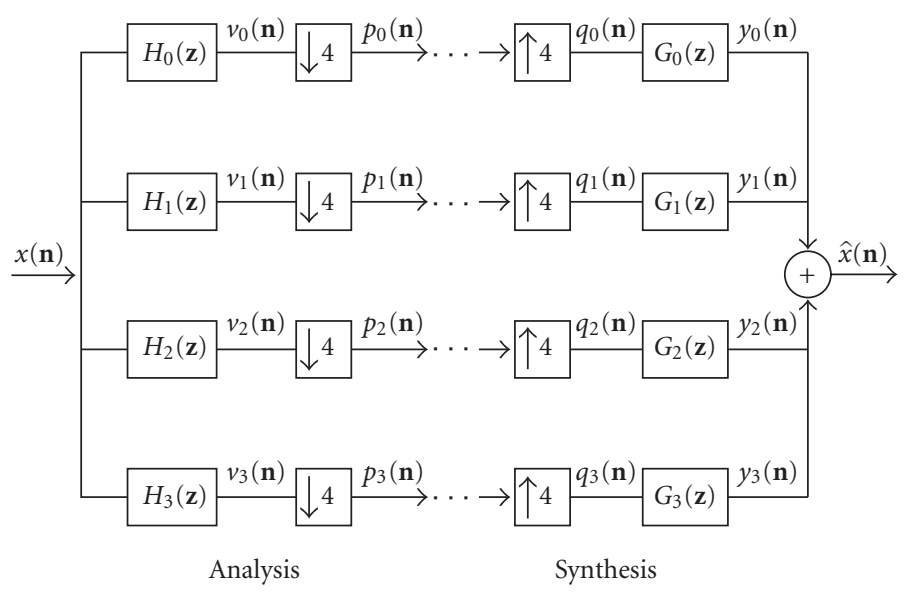

FIgURE 1: Two-dimensional four-channel filter bank.

structures. The four synthesis filters are also combined into a single lattice filter. The analysis section of the PR filter bank is realized with an orthogonal 2D lattice filter; and the synthesis section, which is also an orthogonal 2D lattice filter, is derived according to the PR conditions.

The 3PLF structure is used for the lattice realization, where each stage consists of three lattice parameters and four prediction error fields. We modified these error fields in such a way that each corresponds to a subband of the four-channel filter bank. The four-band frequency split of the 2D spectrum is shown in Figure 2, where the regions 0, 1, 2, and 3 correspond to LL, HL, HH, and LH subbands, respectively. The 3PLF can be decomposed into four filters whose transfer functions can be arranged to generate these four subband filters. Basically, when one of these filters is defined in terms of lattice parameters, the others can be derived from that filter by simply imposing appropriate delays on it as explained in Section 2. Referring to Figure 2, the ideal four-channel filters, $H_{0}(\mathbf{z}), H_{1}(\mathbf{z}), H_{2}(\mathbf{z}), H_{3}(\mathbf{z})$, have mirror image symmetry about their mutual boundaries, which is equivalent to

$$
\begin{gathered}
H_{1}\left(z_{1}, z_{2}\right)=z_{1}^{-1} H_{0}\left(-z_{1}^{-1}, z_{2}\right), \\
H_{2}\left(z_{1}, z_{2}\right)=z_{1}^{-1} z_{2}^{-1} H_{0}\left(-z_{1}^{-1},-z_{2}^{-1}\right), \\
H_{3}\left(z_{1}, z_{2}\right)=z_{2}^{-1} H_{0}\left(z_{1},-z_{2}^{-1}\right) .
\end{gathered}
$$

If one compares (11) with (10a)-(10d), the analogy between the four-quadrant filters of 3PLF and the four subband filters is very obvious.

Starting with a zero-order filter, four fields are generated from the input signal $X\left(z_{1}, z_{2}\right)$ as

$$
\begin{aligned}
X_{0}^{(0)}\left(z_{1}, z_{2}\right) & =X_{1}^{(0)}\left(z_{1}, z_{2}\right)=X_{2}^{(0)}\left(z_{1}, z_{2}\right) \\
& =X_{3}^{(0)}\left(z_{1}, z_{2}\right)=X\left(z_{1}, z_{2}\right) .
\end{aligned}
$$

Here, the superscripts denote the stage number. The transfer function of the first-order filter, $H_{1}^{(0)}\left(z_{1}, z_{2}\right)$, can be expressed in terms of the lattice parameters, $k_{1}^{(1)}, k_{2}^{(1)}$, and $k_{3}^{(1)}$, which are known from the transfer function of the forward prediction error filter defined for the 3PLF structure (cf. (10a)) as follows:

$$
H_{0}^{(1)}\left(z_{1}, z_{2}\right)=1-k_{1}^{(1)} z_{1}^{-1}-k_{2}^{(1)} z_{1}^{-1} z_{2}^{-1}-k_{3}^{(1)} z_{2}^{-1} .
$$

Defining the remaining filters in a similar way, the inputoutput relationship of the analysis lattice filter is given as

$$
\begin{aligned}
{\left[\begin{array}{l}
X_{0}^{(m+1)}\left(z_{1}, z_{2}\right) \\
X_{1}^{(m+1)}\left(z_{1}, z_{2}\right) \\
X_{2}^{(m+1)}\left(z_{1}, z_{2}\right) \\
X_{3}^{(m+1)}\left(z_{1}, z_{2}\right)
\end{array}\right]=} & {\left[\begin{array}{cccc}
1 & -k_{1}^{(m+1)} & -k_{2}^{(m+1)} & -k_{3}^{(m+1)} \\
k_{1}^{(m+1)} & 1 & -k_{3}^{(m+1)} & k_{2}^{(m+1)} \\
-k_{2}^{(m+1)} & k_{3}^{(m+1)} & 1 & k_{1}^{(m+1)} \\
k_{3}^{(m+1)} & k_{2}^{(m+1)} & -k_{1}^{(m+1)} & 1
\end{array}\right] } \\
& \times\left[\begin{array}{c}
X_{0}^{(m)}\left(z_{1}, z_{2}\right) \\
z_{1}^{-1} X_{1}^{(m)}\left(z_{1}, z_{2}\right) \\
z_{1}^{-1} z_{2}^{-1} X_{2}^{(m)}\left(z_{1}, z_{2}\right) \\
z_{2}^{-1} X_{3}^{(m)}\left(z_{1}, z_{2}\right)
\end{array}\right],
\end{aligned}
$$

where $m=0,1, \ldots, M-1$. Compactly, (14) can be expressed as

$$
\mathbf{X}^{(m+1)}\left(z_{1}, z_{2}\right)=\mathbf{H}^{(m+1)} \mathbf{Z} \mathbf{X}^{(m)}\left(z_{1}, z_{2}\right)
$$

where $\mathbf{Z}=\operatorname{diag}\left\{1, z_{1}^{-1}, z_{1}^{-1} z_{2}^{-1}, z_{2}^{-1}\right\}$ and it stands for the delay matrix. Here, it is useful to note that $\mathbf{H}^{(m+1)} \mathbf{Z}=$ $\mathbf{H}^{(m+1)}\left(z_{1}, z_{2}\right)$ and this will be referred to as the transfer matrix of the analysis filter. Defining $\hat{\mathbf{H}}\left(z_{1}, z_{2}\right)$ as the product of the $(m+1)$ transfer functions, namely, 


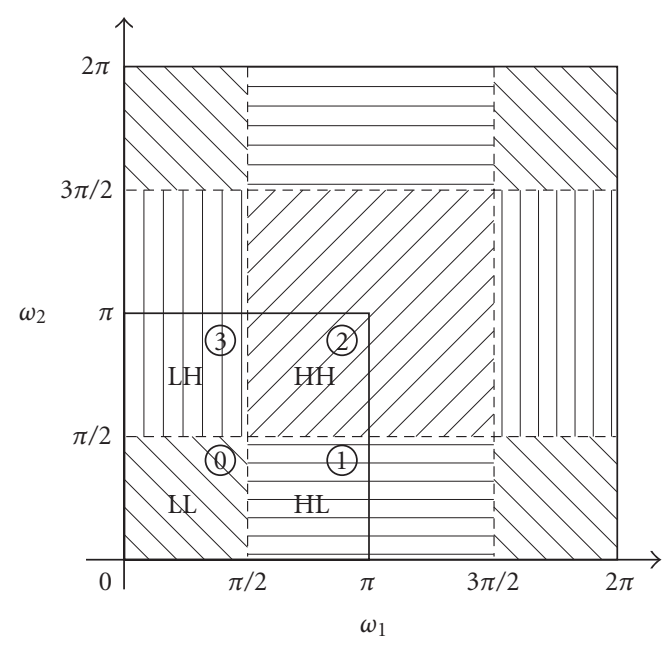

FIGURE 2: Four-band split of 2D spectrum.

$\hat{\mathbf{H}}\left(z_{1}, z_{2}\right)=\left[\mathbf{H}^{(m+1)}\left(z_{1}, z_{2}\right)\right]\left[\mathbf{H}^{(m)}\left(z_{1}, z_{2}\right)\right] \cdots\left[\mathbf{H}^{(1)}\left(z_{1}, z_{2}\right)\right]$, the input-output relation of the complete structure can be given as

$$
\mathbf{X}^{(m+1)}\left(z_{1}, z_{2}\right)=\hat{\mathbf{H}}\left(z_{1}, z_{2}\right) \mathbf{X}^{(0)}\left(z_{1}, z_{2}\right)
$$

The structure of the complete analysis filter characterized by $\hat{\mathbf{H}}\left(z_{1}, z_{2}\right)$ is shown in Figure 3.

\subsection{PR requirements for the analysis and the synthesis lattice filters using polyphase representation}

The 2D lattice filter structure summarized by (14) is nonseparable and the synthesis filter bank has to be determined according to the PR conditions. For the solution of the PR problem, several techniques have been presented however; the most suitable one is the 2D polyphase representation [15] (see Figure 4) which reduces the computational complexity.

The complete analysis lattice filter structure obtained by cascading $(m+1)$ stages will be considered as a single block and the resulting transfer matrix $\hat{\mathbf{H}}\left(z_{1}, z_{2}\right)$ will be viewed as the analysis polyphase matrix, $\mathbf{H}_{p}(\mathbf{z})$. According to the polyphase representation [15], the PR property, denoted by $\mathbf{P}(\mathbf{z})$, is satisfied if

$$
\mathbf{P}(\mathbf{z})=\mathbf{H}_{p}(\mathbf{z}) \mathbf{G}_{p}^{T}(\mathbf{z})=\mathbf{z}^{-\mathbf{n}_{0}} \mathbf{I},
$$

where $\mathbf{G}_{p}(\mathbf{z})$ is the synthesis polyphase filter. This relation can be utilized for the $2 \mathrm{D}$ lattice filter structure as

$$
\mathbf{P}(\mathbf{z})=\hat{\mathbf{H}}\left(z_{1}, z_{2}\right) \hat{\mathbf{G}}\left(z_{1}, z_{2}\right)=\mathbf{z}^{-\mathbf{n}_{0}} \mathbf{I}
$$

where $\hat{\mathbf{G}}\left(z_{1}, z_{2}\right)$ is the transfer function of the complete synthesis filter.

One way to ensure the stability of the synthesis filters is to force the analysis transfer matrix to be paraunitary, that is, $\hat{\mathbf{H}}\left(z_{1}, z_{2}\right) \hat{\mathbf{H}}^{T}\left(z_{1}^{-1}, z_{1}^{-1}\right)=\mathbf{I}$. Then the solution for the synthesis transfer matrix is

$$
\widehat{\mathbf{G}}\left(z_{1}, z_{2}\right)=\mathbf{z}^{-\mathbf{n}_{0}} \hat{\mathbf{H}}^{T}\left(z_{1}^{-1}, z_{2}^{-1}\right) .
$$

The paraunitary condition of transfer matrix at the $i$ th stage of the analysis bank ensures that the transfer matrix of the cascaded lattice structure will also be paraunitary. Thus, the problem reduces to the design of an orthogonal matrix for each stage $i$ such that

$$
\mathbf{H}^{(i)} \mathbf{H}^{(i)}{ }^{T}=\mathbf{I} .
$$

Equation (20) can be written in terms of the lattice filter coefficients as

$$
\mathbf{H}^{(i)} \mathbf{H}^{(i)^{T}}=\left[\begin{array}{cccc}
1+k_{1}^{2}+k_{2}^{2}+k_{3}^{2} & 0 & -2 k_{2}-2 k_{1} k_{3} & 0 \\
0 & 1+k_{1}^{2}+k_{2}^{2}+k_{3}^{2} & 0 & 2 k_{2}+2 k_{1} k_{3} \\
-2 k_{2}-2 k_{1} k_{3} & 0 & 1+k_{1}^{2}+k_{2}^{2}+k_{3}^{2} & 0 \\
0 & 2 k_{2}+2 k_{1} k_{3} & 0 & 1+k_{1}^{2}+k_{2}^{2}+k_{3}^{2}
\end{array}\right] \text {, }
$$




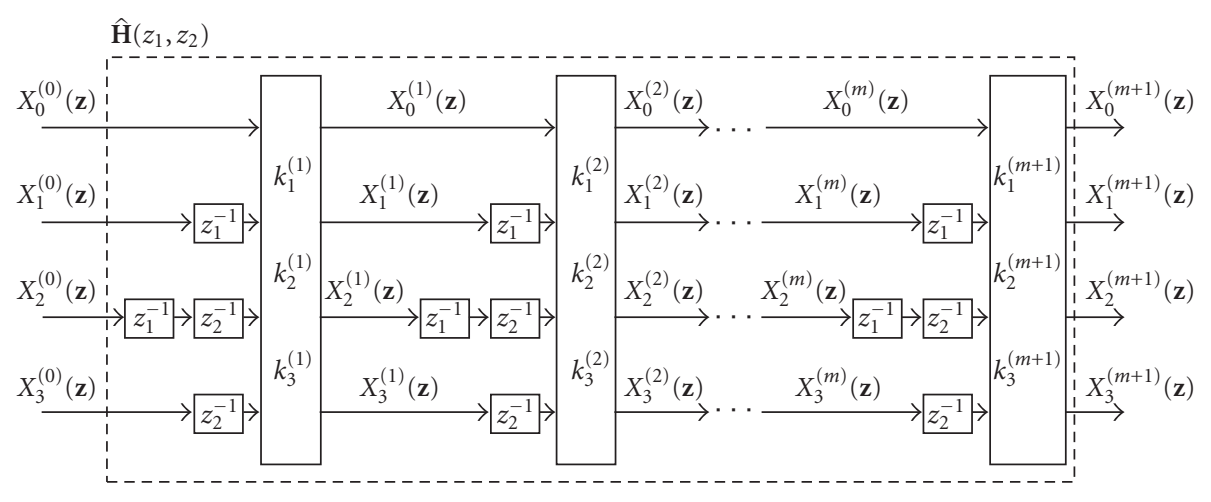

FIGURE 3: The analysis lattice filter, $\hat{\mathbf{H}}\left(z_{1}, z_{2}\right)$.

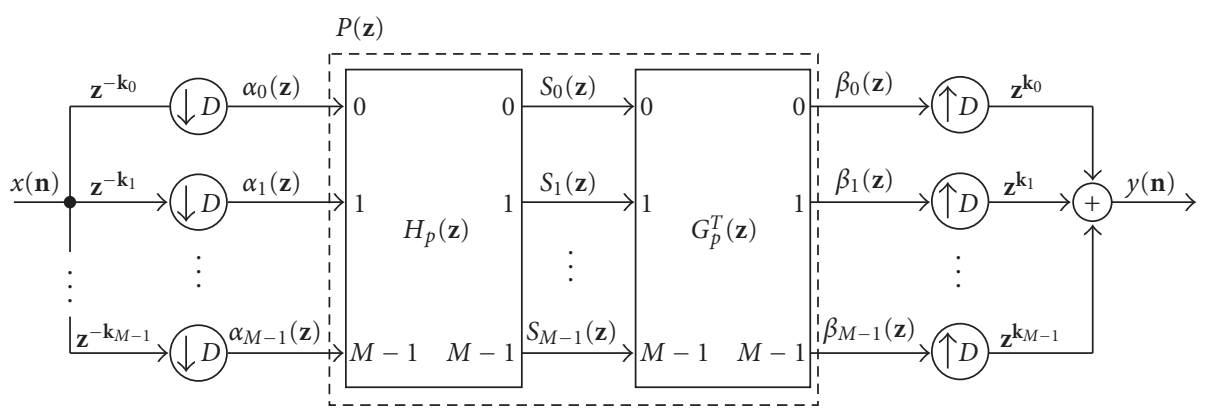

FIGURE 4: Polyphase representation of the analysis and the synthesis filters.

where $k_{j}=k_{j}^{(i)}$ for $j=1,2,3$. Equation (21) can be expressed as a scaled identity matrix if the following constraint is imposed:

$$
k_{2}^{(i)}=-k_{1}^{(i)} k_{3}^{(i)}
$$

In order to obtain an identity matrix solution for (21), each parameter for the $i$ th stage should be divided by a normalizing factor $\delta^{(i)}=\left(1+\left(k_{1}^{(i)}\right)^{2}+\left(k_{2}^{(i)}\right)^{2}+\left(k_{3}^{(i)}\right)^{2}\right)^{1 / 2}$. As a result, the paraunitary condition for the analysis transfer function is satisfied with the constraint defined by (22) and the $\mathrm{PR}$ is possible using the proposed orthogonal lattice filter structure. It is obvious here that the synthesis filter is a rearrangement of the analysis filter. The PR requirement for the synthesis filter given by (19) can be expanded as a product of lower-order filters as

$$
\begin{aligned}
\hat{\mathbf{G}}\left(z_{1}, z_{2}\right)=\mathbf{z}^{-\mathbf{n}_{0}} & {\left[\left(\mathbf{Z}^{-1} \mathbf{H}^{(1)^{T}}\right)\left(\mathbf{Z}^{-1} \mathbf{H}^{(2)^{T}}\right)\right.} \\
& \left.\times\left(\mathbf{Z}^{-1} \mathbf{H}^{(3)^{T}}\right) \cdots\left(\mathbf{Z}^{-1} \mathbf{H}^{(m+1)^{T}}\right)\right] .
\end{aligned}
$$

Hence, the synthesis filter is composed of lattice stages which are the backward arrangements of the analysis lattice stages. Defining the overall delay vector in (23) as $\mathbf{n}_{0}=(m+1) \mathbf{p}_{0}$, the transfer function of each synthesis lattice stage can be given as

$$
\mathbf{G}^{(i)}\left(z_{1}, z_{2}\right)=\mathbf{z}^{-\mathbf{p}_{0}} \mathbf{Z}^{-1} \mathbf{H}^{(i)^{T}}
$$

for $i=1,2, \ldots, m+1$. Selecting $\mathbf{p}_{0}=\left[\begin{array}{ll}1 & 1\end{array}\right]$, one has $\mathbf{z}^{-\mathbf{p}_{0}}=$ $z_{1}^{-1} z_{2}^{-1}$ and the transfer function for order $(m+1)$ can be explicitly written as

$$
\begin{aligned}
\mathbf{G}^{(m+1)}\left(z_{1}, z_{2}\right)= & {\left[\begin{array}{cccc}
z_{1}^{-1} z_{2}^{-1} & 0 & 0 & 0 \\
0 & z_{2}^{-1} & 0 & 0 \\
0 & 0 & 1 & 0 \\
0 & 0 & 0 & z_{1}^{-1}
\end{array}\right] } \\
& \times\left[\begin{array}{cccc}
1 & k_{1}^{(m+1)} & -k_{2}^{(m+1)} & k_{3}^{(m+1)} \\
-k_{1}^{(m+1)} & 1 & k_{3}^{(m+1)} & k_{2}^{(m+1)} \\
-k_{2}^{(m+1)} & -k_{3}^{(m+1)} & 1 & -k_{1}^{(m+1)} \\
-k_{3}^{(m+1)} & k_{2}^{(m+1)} & k_{1}^{(m+1)} & 1
\end{array}\right] .
\end{aligned}
$$

The input-output relation for the synthesis lattice filter can be given as

$$
\mathbf{Y}^{(0)}\left(z_{1}, z_{2}\right)=\hat{\mathbf{G}}\left(z_{1}, z_{2}\right) \mathbf{Y}^{(m+1)}\left(z_{1}, z_{2}\right)
$$

The complete synthesis lattice filter structure is characterized by the transfer function $\widehat{\mathbf{G}}\left(z_{1}, z_{2}\right)$. Its block diagram can be obtained from that of the analysis filter by arranging the stages in a backward fashion and changing the delays appropriately. 


\subsection{NSPRL filter bank realized with the orthogonal $2 D$ lattice filter structure}

An alternative polyphase representation can be obtained when $\hat{\mathbf{H}}(\mathbf{z})$ is carried to the left-hand side of the decimators and $\widehat{\mathbf{G}}(\mathbf{z})$ is carried to the right-hand side of the interpolators as in the configuration shown in Figure 5 [15]. In this case, according to the quadratic-sampling scheme, the arguments of the transfer functions should be replaced by $\mathbf{z}^{\mathbf{D}}=z_{1}^{2} z_{2}^{2}$. In other words, the transfer matrices of the analysis and the synthesis lattice models, $\mathbf{H}^{(2 m)}\left(z_{1}, z_{2}\right)$ and $\mathbf{G}^{(2 m)}\left(z_{1}, z_{2}\right)$, should be replaced with their counterparts, $\mathbf{H}^{(2 m)}\left(z_{1}^{2}, z_{2}^{2}\right)$ and $\mathbf{G}^{(2 m)}\left(z_{1}^{2}, z_{2}^{2}\right)$, respectively, as follows:

$$
\begin{aligned}
\mathbf{H}^{(2 m)}\left(z_{1}^{2}, z_{2}^{2}\right)= & {\left[\begin{array}{cccc}
1 & -k_{1}^{(2 m)} & -k_{2}^{(2 m)} & -k_{3}^{(2 m)} \\
k_{1}^{(2 m)} & 1 & -k_{3}^{(2 m)} & k_{2}^{(2 m)} \\
-k_{2}^{(2 m)} & k_{3}^{(2 m)} & 1 & k_{1}^{(2 m)} \\
k_{3}^{(2 m)} & k_{2}^{(2 m)} & -k_{1}^{(2 m)} & 1
\end{array}\right] } \\
& \times\left[\begin{array}{cccc}
1 & 0 & 0 & 0 \\
0 & z_{1}^{-2} & 0 & 0 \\
0 & 0 & z_{1}^{-2} z_{2}^{-2} & 0 \\
0 & 0 & 0 & z_{2}^{-2}
\end{array}\right], \\
\mathbf{G}^{(2 m)}\left(z_{1}^{2}, z_{2}^{2}\right)= & {\left[\begin{array}{cccc}
z_{1}^{-2} z_{2}^{-2} & 0 & 0 & 0 \\
0 & z_{2}^{-2} & 0 & 0 \\
0 & 0 & 1 & 0 \\
0 & 0 & 0 & z_{1}^{-2}
\end{array}\right] } \\
& \times\left[\begin{array}{cccc}
1 & k_{1}^{(2 m)} & -k_{2}^{(2 m)} & k_{3}^{(2 m)} \\
-k_{1}^{(2 m)} & 1 & k_{3}^{(2 m)} & k_{2}^{(2 m)} \\
-k_{2}^{(2 m)} & -k_{3}^{(2 m)} & 1 & -k_{1}^{(2 m)} \\
-k_{3}^{(2 m)} & k_{2}^{(2 m)} & k_{1}^{(2 m)} & 1
\end{array}\right] .
\end{aligned}
$$

When the analysis and the synthesis lattice filters are moved to obtain the proposed NSPRL structure in the form given in Figure 5 , the delay operators $\left(1, z_{1}, z_{1} z_{2}, z_{2}\right)$ at the beginning of the modified analysis polyphase section and those $\left(z_{1} z_{2}, z_{2}, 1, z_{1}\right)$ at the end of the modified synthesis polyphase section are multiplied with the delay operators of the first stages of the analysis and the synthesis lattice filters which are $\left(1, z_{1}^{-2}, z_{1}^{-2} z_{2}^{-2}, z_{2}^{-2}\right)$ and $\left(z_{1}^{-2} z_{2}^{-2}, z_{2}^{-2}, 1, z_{1}^{-2}\right)$, respectively. The result of this operation gives the sets of delay operators $\left(1, z_{1}^{-1}, z_{1}^{-1} z_{2}^{-1}, z_{2}^{-1}\right)$ and $\left(z_{1}^{-1} z_{2}^{-1}, z_{2}^{-1}, 1, z_{1}^{-1}\right)$ only for the first stages of these two lattice realizations.

Since the lattice stages, except the first stage, contain the squares of the delay operators, they can be interpreted as a pair of stages, the first of which has zero lattice parameters. Therefore, the NSPRL structure consists of odd-numbered stages and the word "order" is used to refer to the total number of delays introduced up to the last stage of the lattice filter structure.

\section{The analysis section of the NSPRL filter bank}

The input-output relations for the first and the odd-numbered orders of the analysis filter can be given as

$$
\begin{aligned}
{\left[\begin{array}{l}
X_{0}^{(p)}\left(z_{1}, z_{2}\right) \\
X_{1}^{(p)}\left(z_{1}, z_{2}\right) \\
X_{2}^{(p)}\left(z_{1}, z_{2}\right) \\
X_{3}^{(p)}\left(z_{1}, z_{2}\right)
\end{array}\right]=} & {\left[\begin{array}{cccc}
1 & -k_{1}^{(p)} & -k_{2}^{(p)} & -k_{3}^{(p)} \\
k_{1}^{(p)} & 1 & -k_{3}^{(p)} & k_{2}^{(p)} \\
-k_{2}^{(p)} & k_{3}^{(p)} & 1 & k_{1}^{(p)} \\
k_{3}^{(p)} & k_{2}^{(p)} & -k_{1}^{(p)} & 1
\end{array}\right] } \\
& \times\left[\begin{array}{c}
X_{0}^{(r)}\left(z_{1}, z_{2}\right) \\
z_{1}^{-q} X_{1}^{(r)}\left(z_{1}, z_{2}\right) \\
z_{1}^{-q} z_{2}^{-q} X_{2}^{(r)}\left(z_{1}, z_{2}\right) \\
z_{2}^{-q} X_{3}^{(r)}\left(z_{1}, z_{2}\right)
\end{array}\right] .
\end{aligned}
$$

In (28), $p=1, q=1$, and $r=0$ for the first order and $p=(2 m+1), q=2$, and $r=(2 m-1)$ for the odd-numbered orders where $m=(1,2, \ldots, M)$. Note that the constraints $k_{2}^{(p)}=-k_{1}^{(p)} k_{3}^{(p)}$ are to be imposed for orthogonality. The analysis lattice filter structure is illustrated in Figure 6.

\section{The synthesis section of the NSPRL filter bank}

The input-output relations for the first and the odd-numbered orders of the synthesis filter are given as

$$
\begin{aligned}
{\left[\begin{array}{c}
Y_{0}^{(r)}\left(z_{1}, z_{2}\right) \\
Y_{1}^{(r)}\left(z_{1}, z_{2}\right) \\
Y_{2}^{(r)}\left(z_{1}, z_{2}\right) \\
Y_{3}^{(r)}\left(z_{1}, z_{2}\right)
\end{array}\right] } & {\left[\begin{array}{cccc}
z_{1}^{-q} z_{2}^{-q} & 0 & 0 & 0 \\
0 & z_{2}^{-q} & 0 & 0 \\
0 & 0 & 1 & 0 \\
0 & 0 & 0 & z_{1}^{-q}
\end{array}\right] } \\
& \times\left[\begin{array}{cccc}
1 & k_{1}^{(p)} & -k_{2}^{(p)} & k_{3}^{(p)} \\
-k_{1}^{(p)} & 1 & k_{3}^{(p)} & k_{2}^{(p)} \\
-k_{2}^{(p)} & -k_{3}^{(p)} & 1 & -k_{1}^{(p)} \\
-k_{3}^{(p)} & k_{2}^{(p)} & k_{1}^{(p)} & 1
\end{array}\right] \\
& \times\left[\begin{array}{c}
Y_{0}^{(p)}\left(z_{1}, z_{2}\right) \\
Y_{1}^{(p)}\left(z_{1}, z_{2}\right) \\
Y_{2}^{(p)}\left(z_{1}, z_{2}\right) \\
Y_{3}^{(p)}\left(z_{1}, z_{2}\right)
\end{array}\right] .
\end{aligned}
$$

In (29), $r=(2 m-1), q=2$, and $p=(2 m+1)$ for the oddnumbered orders where for $m=(M, \ldots, 2,1)$ and $r=0$, $q=1$ and $p=1$ for the first order. The constraints are given as $k_{2}^{(p)}=-k_{1}^{(p)} k_{3}^{(p)}$. The synthesis lattice filter structure is shown in Figure 7 . Here the output $y(\mathbf{n})$ is the estimate $\hat{x}(\mathbf{n})$ of the original input signal $x(\mathbf{n})$. 


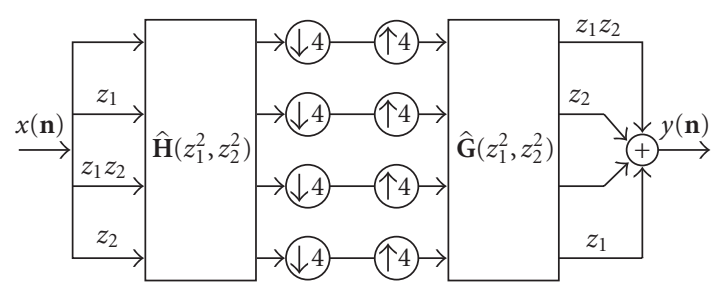

FIGURE 5: Modified polyphase representation for NSPRL.

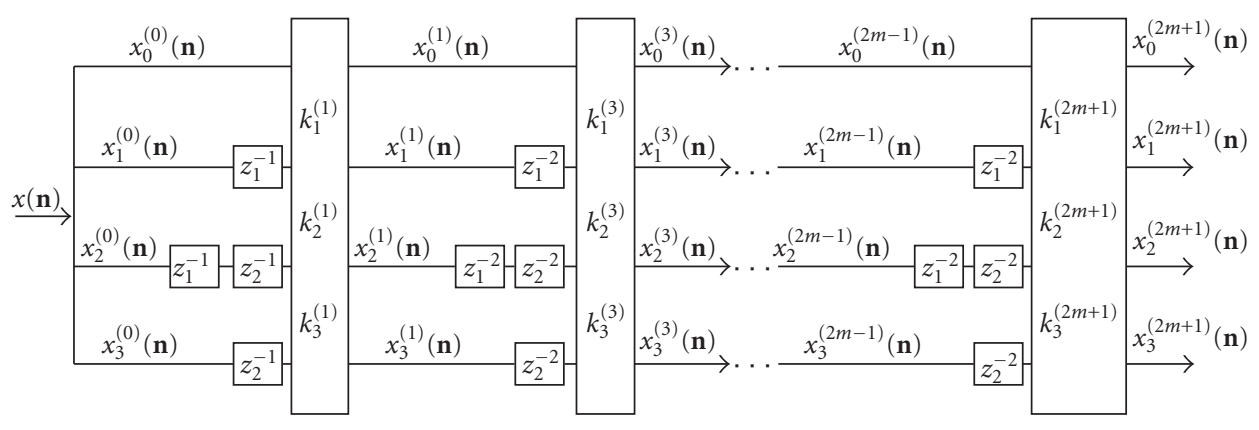

FIgURE 6: The analysis section of the NSPRL filter bank.

\subsection{Computation of the lattice coefficients}

Once the analysis and the synthesis filter structures are obtained, the next step is to determine the lattice coefficients at each stage using an optimization algorithm. The optimization algorithm developed here depends on the determination of the stopband edges and the minimization of the stopband energy in the 2D frequency domain. Although the analysis lattice filter consists of four filters at each stage, it is sufficient to consider only one of them since the others are shifted versions of it.

The desired frequency response of any of the four subband filters can be used to determine the stopband energy. Taking $H_{2}^{(2 m+1)}$ as the $\mathrm{HH}$ filter, the objective is to minimize the energy content outside region 2 shown in Figure 2. That is,

$$
\mathbf{E}^{(2 m+1)}=\iint_{\left(\omega_{1}, \omega_{2}\right) \in R_{2}^{\prime}}\left|\mathbf{H}_{2}^{(2 m+1)}\left(e^{j \omega_{1}}, e^{j \omega_{2}}\right)\right|^{2} d \omega_{1} d \omega_{2},
$$

where $R_{2}^{\prime}=\left\{0 \leq\left(\omega_{1}, \omega_{2}\right) \leq \pi,\left(\omega_{1}, \omega_{2}\right) \notin R_{2}\right\}$. The four subband regions shown in Figure 2 are usually designed for the ideal subband decomposition systems. Region 2, for example, covers the frequency spectrum $[\pi / 2 \rightarrow \pi, \pi / 2 \rightarrow \pi]$ having a square-shape characteristic. However, it is possible to adjust these stopband edges as $\left[\pi / 2 \pm \varepsilon_{1}, \pi / 2 \pm \varepsilon_{2}\right]$ according to the desired frequency specifications.

In $(30)$, the transfer function of the $(2 m+1)$ th-order filter can be written in terms of those of the $(2 m-1)$ th-order filters using (28) as follows. (i) For the first-order stage,

$$
\begin{aligned}
& H_{2}^{(1)}\left(e^{j \omega_{1}}, e^{j \omega_{2}}\right) \\
& \quad=\frac{1}{\delta^{(1)}}\left[-k_{2}^{(1)}+k_{3}^{(1)} e^{-j \omega_{1}}+e^{-j \omega_{1}} e^{-j \omega_{2}}+k_{1}^{(1)} e^{-j \omega_{2}}\right]
\end{aligned}
$$

since

$$
\begin{aligned}
H_{0}^{(0)}\left(e^{j \omega_{1}}, e^{j \omega_{2}}\right) & =H_{1}^{(0)}\left(e^{j \omega_{1}}, e^{j \omega_{2}}\right)=H_{2}^{(0)}\left(e^{j \omega_{1}}, e^{j \omega_{2}}\right) \\
& =H_{3}^{(0)}\left(e^{j \omega_{1}}, e^{j \omega_{2}}\right)=1
\end{aligned}
$$

for all $\left(\omega_{1}, \omega_{2}\right)$.

(ii) For the odd-numbered order stages,

$$
\begin{aligned}
H_{2}^{(2 m+1)}\left(e^{j \omega_{1}}, e^{j \omega_{2}}\right)= & \frac{1}{\delta^{(2 m+1)}} \\
& \times\left[-k_{2}^{(2 m+1)} H_{0}^{(2 m-1)}\left(e^{j 2 \omega_{1}}, e^{j 2 \omega_{2}}\right)\right. \\
& +k_{3}^{(2 m+1)} e^{-j 2 \omega_{1}} H_{1}^{(2 m-1)}\left(e^{j 2 \omega_{1}}, e^{j 2 \omega_{2}}\right) \\
& +e^{-j 2 \omega_{1}} e^{-j 2 \omega_{2}} H_{2}^{(2 m-1)}\left(e^{j 2 \omega_{1}}, e^{j 2 \omega_{2}}\right) \\
& \left.+k_{1}^{(2 m+1)} e^{-j 2 \omega_{2}} H_{3}^{(2 m-1)}\left(e^{j 2 \omega_{1}}, e^{j 2 \omega_{2}}\right)\right],
\end{aligned}
$$

where $(m=1,2, \ldots, M)$ and $\delta^{(2 m+1)}$ is the normalization constant defined previously. The magnitude squared term in 


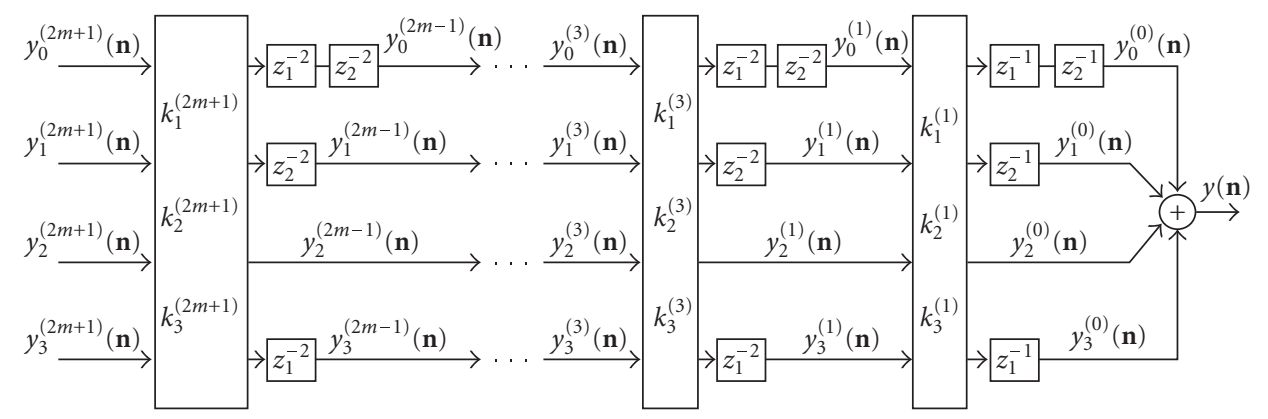

FIgURE 7: The synthesis section of the NSPRL filter bank.

(30) can be calculated for the odd-numbered orders as

$$
\begin{aligned}
\left|H_{2}^{(2 m+1)}\right|^{2}= & H_{2}^{(2 m+1)} H_{2}^{(2 m+1)^{*}} \\
= & k_{2}^{2} H_{0} H_{0}^{*}+k_{3}^{2} H_{1} H_{1}^{*}+H_{2} H_{2}^{*}+k_{1}^{2} H_{3} H_{3}^{*} \\
& +k_{1}\left(e^{j 2 \omega_{1}} H_{3} H_{2}^{*}+e^{-j 2 \omega_{1}} H_{3}^{*} H_{2}\right) \\
& -k_{2}\left(e^{j 2 \omega_{1}} e^{j 2 \omega_{2}} H_{0} H_{2}^{*}+e^{-j 2 \omega_{1}} e^{-j 2 \omega_{2}} H_{0}^{*} H_{2}\right) \\
& +k_{3}\left(e^{j 2 \omega_{2}} H_{1} H_{2}^{*}+e^{-j 2 \omega_{2}} H_{1}^{*} H_{2}\right) \\
& -k_{1} k_{2}\left(e^{j 2 \omega_{2}} H_{0} H_{3}^{*}+e^{-j 2 \omega_{2}} H_{0}^{*} H_{3}\right) \\
& +k_{1} k_{3}\left(e^{-j 2 \omega_{1}} e^{j 2 \omega_{2}} H_{1} H_{3}^{*}+e^{j 2 \omega_{1}} e^{-j 2 \omega_{2}} H_{1}^{*} H_{3}\right) \\
& \left.-k_{2} k_{3}\left(e^{-j 2 \omega_{1}} H_{1} H_{0}^{*}+e^{j 2 \omega_{1}} H_{1}^{*} H_{0}\right)\right] \\
& \times \frac{1}{\left(1+k_{1}^{2}+k_{2}^{2}+k_{3}^{2}\right)} .
\end{aligned}
$$

For clarity, the superscripts, $(2 m-1)$, that denote the orders of the transfer functions as well as the lattice parameters, and the arguments $\left(e^{j 2 \omega_{1}}, e^{j 2 \omega_{2}}\right)$ in (34) are omitted. The same equation can be used for the first-order lattice filter by replacing the transfer functions with unity (see (32)) and dropping the squares in exponential terms.

The lattice coefficients can be computed by equating the gradient of the objective function with respect to the lattice parameters to zero.

\subsection{Extension of the 1D two-channel PRL filter to the 2D four channel separable PRL filter and the relationship with the proposed NSPRL filter}

Here, the 1D two-channel PR lattice filter structure developed by Vaidyanathan and Hoang [14] is extended to 2D in order to obtain a 2D four-channel SPRL filter bank.

The lattice filter realization in [14] splits the $1 \mathrm{D}$ frequency spectrum into an $\mathrm{L}$ and an $\mathrm{H}$ pass component. As in the case of separable filters, a second split can be applied to these $\mathrm{L}$ and $\mathrm{H}$ subbands to obtain LL, LH, HH, and HL subbands in 2D. In general, a 2D filter is said to be separable in the $z$-domain if its transfer function can be written as a product of two 1D transfer functions. Hence, the transfer functions of the filters for the four-band split of a $2 \mathrm{D}$ signal can be written in terms of $1 \mathrm{D}$ filters as

$$
\begin{gathered}
H_{\mathrm{LL}}\left(z_{1}, z_{2}\right)=H_{\mathrm{L}}\left(z_{1}\right) H_{L}\left(z_{2}\right), \\
H_{\mathrm{LH}}\left(z_{1}, z_{2}\right)=H_{\mathrm{L}}\left(z_{1}\right) H_{\mathrm{H}}\left(z_{2}\right), \\
H_{\mathrm{HH}}\left(z_{1}, z_{2}\right)=H_{\mathrm{H}}\left(z_{1}\right) H_{\mathrm{H}}\left(z_{2}\right), \\
H_{\mathrm{HL}}\left(z_{1}, z_{2}\right)=H_{\mathrm{H}}\left(z_{1}\right) H_{\mathrm{L}}\left(z_{2}\right) .
\end{gathered}
$$

The transfer functions of the $\mathrm{L}$ and $\mathrm{H}$ filters of order 1 can be, respectively, given as

$$
\begin{gathered}
H_{\mathrm{L}}^{(1)}(z)=1-\alpha_{1} z^{-1}, \\
H_{\mathrm{H}}^{(1)}(z)=\alpha_{1}+z^{-1},
\end{gathered}
$$

where $\alpha_{1}$ is the lattice filter coefficient for the 1D first-order filter.

The transfer functions for the separable LL, HL, HH, and LH filters can be expressed easily using (35) and (36). It should be noted that only the odd-ordered stages exist. The input-output relation of the separable 2D filter for stage $(2 m+1)$ is defined as follows:

$$
\begin{aligned}
{\left[\begin{array}{l}
X_{0}^{(2 m+1)}\left(z_{1}, z_{2}\right) \\
X_{1}^{(2 m+1)}\left(z_{1}, z_{2}\right) \\
X_{2}^{(2 m+1)}\left(z_{1}, z_{2}\right) \\
X_{3}^{(2 m+1)}\left(z_{1}, z_{2}\right)
\end{array}\right]=} & {\left[\begin{array}{cccc}
1 & -\alpha_{2 m+1} & \alpha_{2 m+1}^{2} & -\alpha_{2 m+1} \\
\alpha_{2 m+1} & 1 & -\alpha_{2 m+1} & -\alpha_{2 m+1}^{2} \\
\alpha_{2 m+1}^{2} & \alpha_{2 m+1} & 1 & \alpha_{2 m+1} \\
\alpha_{2 m+1} & -\alpha_{2 m+1}^{2} & -\alpha_{2 m+1} & 1
\end{array}\right] } \\
& \times\left[\begin{array}{c}
X_{0}^{(2 m-1)}\left(z_{1}, z_{2}\right) \\
z_{1}^{-2} X_{1}^{(2 m-1)}\left(z_{1}, z_{2}\right) \\
z_{1}^{-2} z_{2}^{-2} X_{2}^{(2 m-1)}\left(z_{1}, z_{2}\right) \\
z_{2}^{-2} X_{3}^{(2 m-1)}\left(z_{1}, z_{2}\right)
\end{array}\right]
\end{aligned}
$$




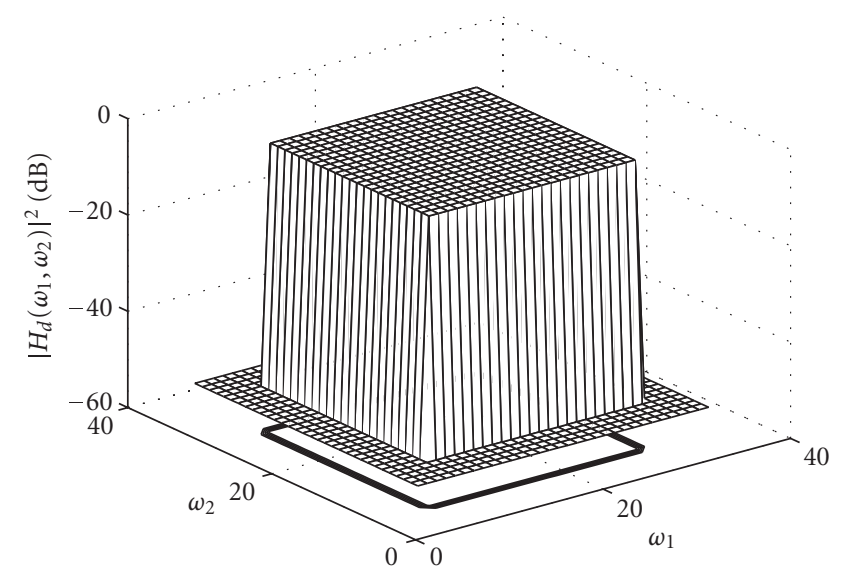

FIGURE 8: Frequency response of the desired HH filter; $\left(\omega_{s_{1}}, \omega_{s_{2}}\right)=(0.22 \pi, 0.22 \pi)$.

Hence, starting from a 1D filter and using the concept of separability, a 2D filter is derived. The structure consists of only one parameter at each stage, which is, in fact, the parameter of the 1D lattice filter. This means that if the calculated lattice parameters lead to a desired pair of $\mathrm{L}$ and $\mathrm{H}$ filters in $1 \mathrm{D}$, then they will also result in the desired LL, HL, HH, and LH filters in 2D.

When (37) is compared with the transfer function of the analysis section of the proposed 2D four-channel NSPRL filter bank obtained by polyphase technique, it is easily seen that the separable structure is a special case of that of the NSPRL under the following conditions:

$$
\begin{gathered}
k_{1}^{(m)}=k_{3}^{(m)}=\alpha_{m}, \\
k_{2}^{(m)}=-\left(\alpha_{m}\right)^{2} .
\end{gathered}
$$

Equation (38b) indicates that the separable lattice filter is orthogonal and satisfies the PR property as in the case of proposed 2D four-channel NSPRL filter where the condition $k_{2}=-k_{1} k_{3}$ is imposed as a constraint. Therefore, all the requirements satisfied by the proposed NSPRL filter structure are also fulfilled by the separable lattice filter structure provided (38a) holds. For the separable filter, this condition is equivalent to the additional constraint that the stopband edge frequencies along both directions should be equal, that is, $\omega_{s_{1}}=\omega_{s_{2}}$; where $\omega_{s_{1}}$ and $\omega_{s_{2}}$ are the stopband frequencies along the horizontal and the vertical directions, respectively, for the separable filter. Only when (38a) holds, the four-quadrant symmetry is satisfied and separable design becomes possible.

\section{COMPUTER SIMULATIONS AND RESULTS}

The computer simulations, carried out with the MATLAB programming language, consist of two parts: the design of the subband filters for different stopband edge frequencies and the processing of a monochrome image, of size $256 \times 256$ with 8-bit gray scale, by these filters to verify the PR property of the proposed structure. Many different simulations are carried out. Two design examples are presented, one involving square filters, the other rectangular filters.

Example 1. In this example, an $\mathrm{HH}$ filter design is considered and the PR property of the proposed NSPRL structure is verified. The desired cut-off frequencies are $\left(\omega_{s_{1}}, \omega_{s_{2}}\right)=$ $(0.22 \pi, 0.22 \pi)$. The specifications on filter characteristics are given in Figure 8. The same procedure is repeated for SPRL and 3 PLF in order to compare their performances. Note that 3PLF is not a PR filter. The reflection coefficients of the proposed method are calculated using the optimization procedure discussed in Section 3.3. In order to design the SPRL filter bank, the 1D reflection coefficients tabulated in [14] are used and the 2D filters are designed according to (38). For the 3PLF, the reflection coefficients are calculated as in Section 3.3 without imposing the constraint $k_{2}=-k_{1} k_{3}$ and without using the normalization factor since these are the requirements for the PR property. All filters are taken to be of order 7. To give the notion of evolution of the frequency responses of the subband filters towards the desired response, the magnitude plots obtained at the end of the first and the seventh orders are shown in Figure 9 for each of the three filter structures. The lattice filter parameters and the stopband average power for each order are also given in Table 1. From Figure 9, it is clearly seen that as the order of the lattice filter structure increases, the transfer function of the $\mathrm{HH}$ filter and therefore those of other subband filters become closer to the desired filter characteristics and sharper subband filters are obtained.

The subband filters are applied to the Lena image and the resulting subband images at the end of the seventh-order filter and the reconstructed images, in the absence of encoding/decoding, are illustrated in Figures 10 and 11, respectively. As observed from the subband images, the 3PLF cannot decompose the original image into its subband components as much as the NSPRL or the SPRL filter banks do. It 

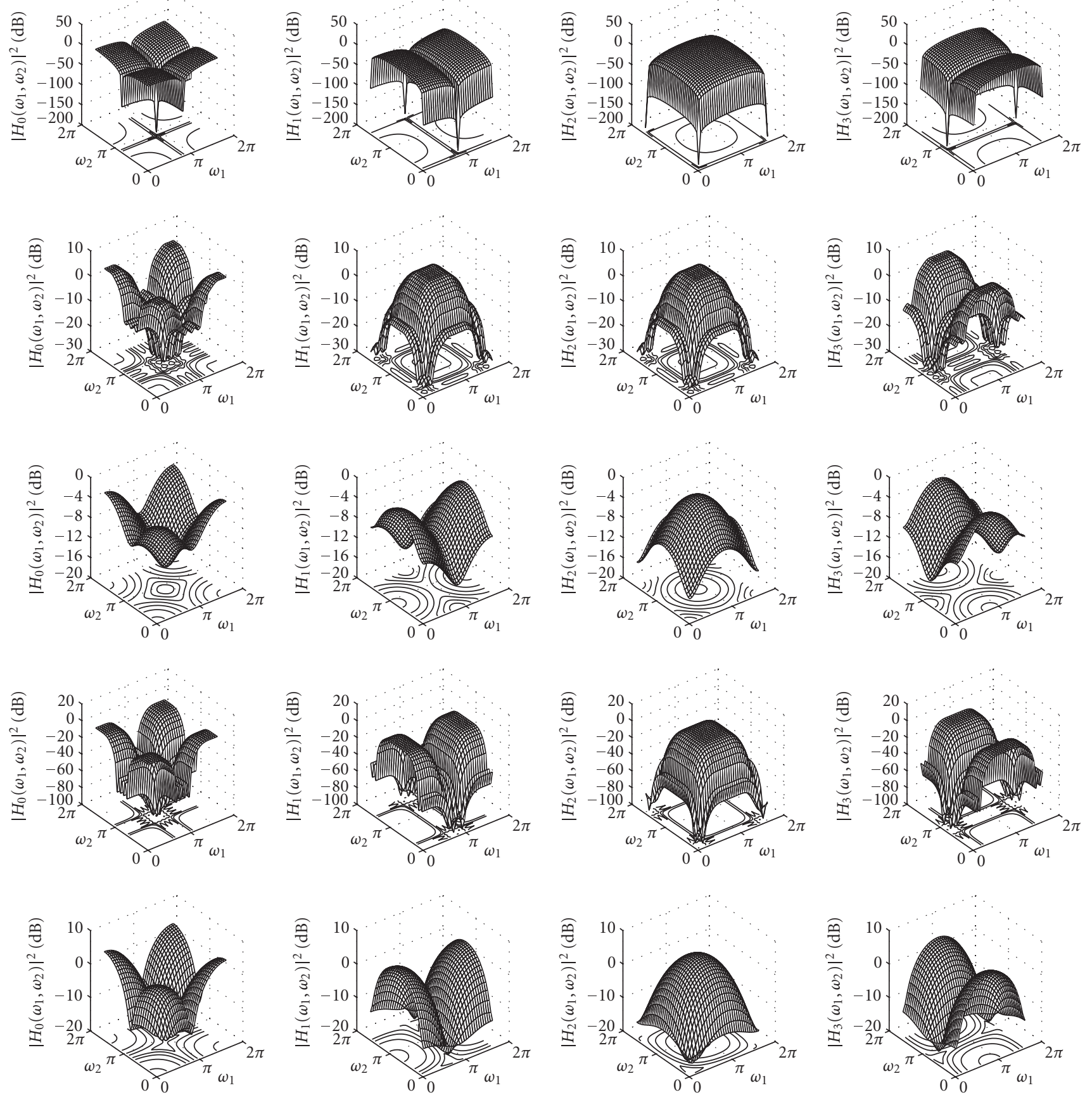

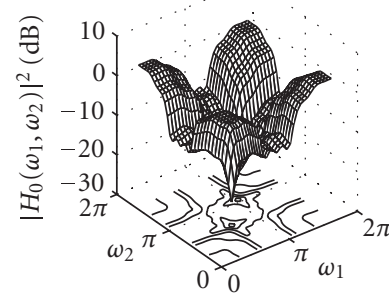

(a)

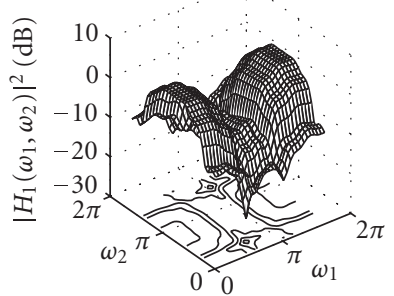

(b)

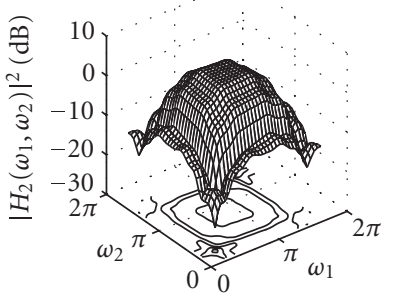

(c)

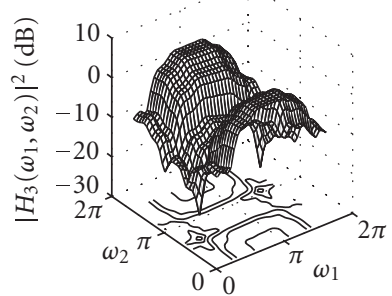

(d)

FIgURE 9: Frequency reponses of the subband filters for (1st row) NSPRL order 1; (2nd row) NSPRL order 7; (3rd row) SPRL order 1; (4th row) SPRL order 7; (5th row) 3PLF order 1; (6th row) 3PLF order 7. Cut-off frequencies for the HH filter are $\left(\omega_{s_{1}}, \omega_{s_{2}}\right)=(0.22 \pi, 0.22 \pi)$. (a) LL filters; (b) HL filters; (c) HH filters; (d) LH filters. 
TABLE 1: Results of the filter design with $\left(\omega_{s_{1}}, \omega_{s_{2}}\right)=(0.22 \pi, 0.22 \pi)$.

\begin{tabular}{c|c|ccc|c}
\hline Filter structure & Order & \multicolumn{3}{|c|}{ Lattice filter coefficients } & Stopband average power \\
\hline & & $k_{1}$ & $k_{2}$ & $k_{3}$ & \\
\hline \multirow{3}{*}{ NSPRL } & 1 & -1 & -1 & -1 & 0.1415 \\
& 3 & 0.1913 & -0.0366 & 0.1913 & 0.0660 \\
& 5 & $0.4229 \mathrm{e}-003$ & $-0.0002 \mathrm{e}-003$ & $0.4229 \mathrm{e}-003$ & 0.0660 \\
& 7 & -0.0426 & -0.0018 & -0.0426 & 0.0621 \\
\hline \multirow{3}{*}{ SPRL } & 1 & -2.6380 & -6.9590 & -2.6380 & 0.1147 \\
& 3 & 0.7154 & -0.5119 & 0.7154 & 0.0312 \\
& 5 & -0.2598 & -0.0675 & -0.2598 & 0.0035 \\
& 7 & 0.0639 & -0.0041 & 0.0639 & 0.0004 \\
\hline \multirow{3}{*}{3 PLF } & 1 & -0.6809 & -0.6247 & -0.6809 & 0.1823 \\
& 3 & 0.1074 & -0.0205 & 0.1074 & 0.1529 \\
& 5 & 0.0496 & -0.0042 & 0.0496 & 0.1466 \\
& 7 & -0.0557 & -0.0042 & -0.0557 & 0.1388 \\
\hline
\end{tabular}
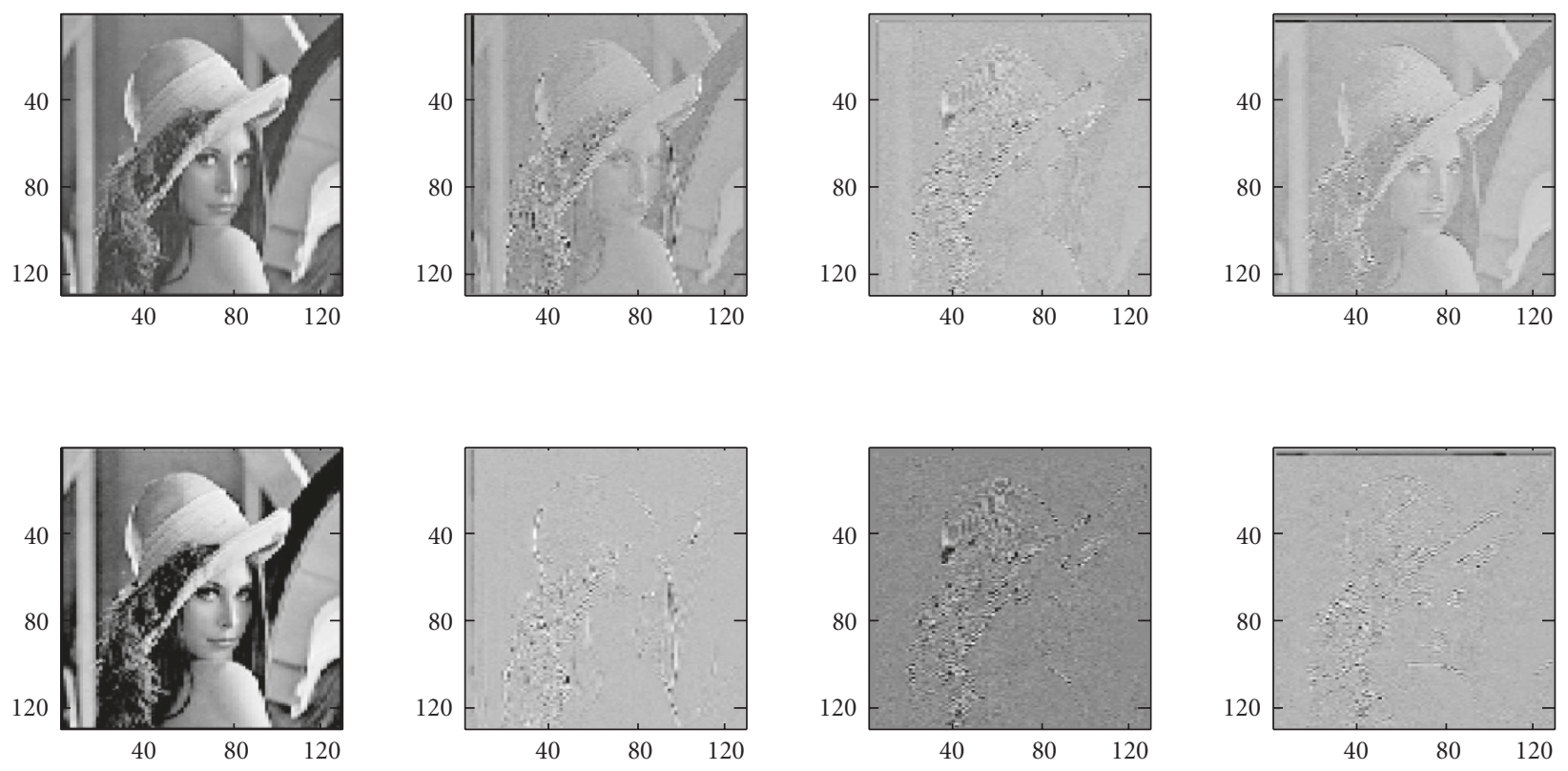

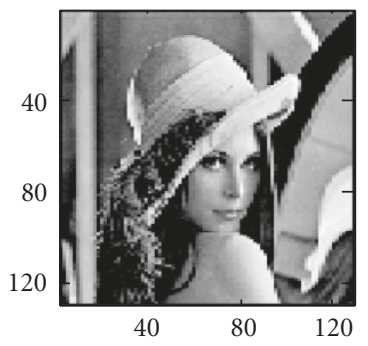

(a)

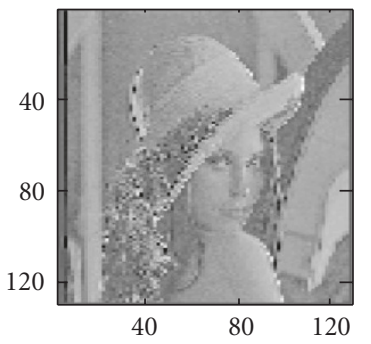

(b)

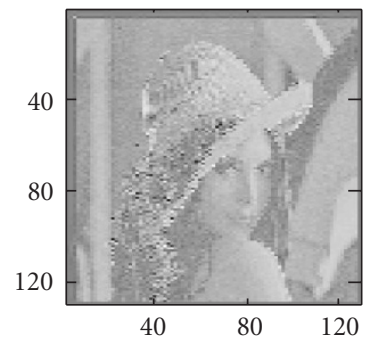

(c)

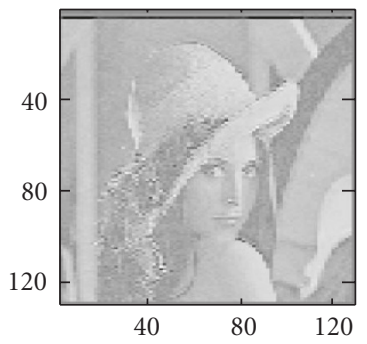

(d)

FIgURE 10: The subimages obtained using (1st row) the 7th-order NSPRL subband filters; (2nd row) 7th-order SPRL subband filters; (3rd row) 7th-order 3PLF subband filters. For $\left(\omega_{s_{1}}, \omega_{s_{2}}\right)=(0.22 \pi, 0.22 \pi)$. (a) LL subimages; (b) HL subimages; (c) HH subimages; (d) LH subimages. 


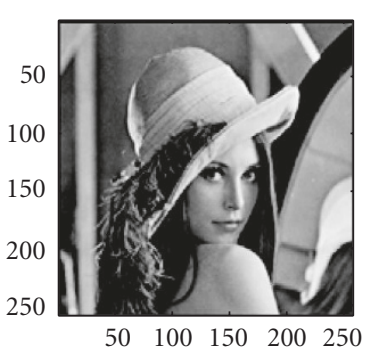

(a)

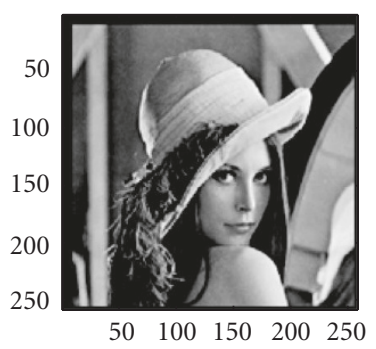

(c)

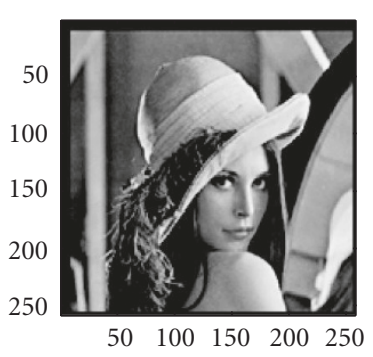

(b)

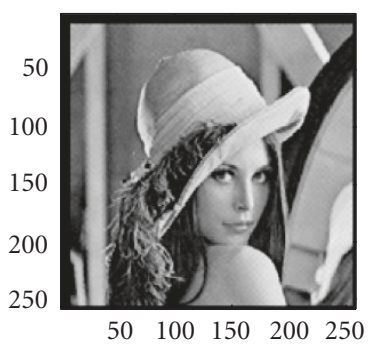

(d)

FIGURE 11: (a) The original image; (b) the reconstructed image using the proposed NSPRL filter bank; (c) the reconstructed image using the SPRL filter bank; (d) the reconstructed image using the $3 \operatorname{PLF}\left(\omega_{s_{1}}, \omega_{s_{2}}\right)=(0.22 \pi, 0.22 \pi)$.

TABLE 2: SNR values related to reconstruction by different filter banks for $\left(\omega_{s_{1}}, \omega_{s_{2}}\right)=(0.22 \pi, 0.22 \pi)$.

\begin{tabular}{cc}
\hline Filter structure & SNR $(\mathrm{dB})$ \\
\hline NSPRL & 90.15 \\
SPRL & 74.61 \\
3PLF & 15.14 \\
\hline
\end{tabular}

is seen that the PR of the Lena image is not possible with the 3PLF as expected. The proposed method and the SPRL filter perfectly reconstruct the image. To judge these images quantitatively, the signal to noise (i.e., reconstruction error) ratio (SNR) is calculated as the ratio of the mean squared value of the original image to that of the error image, where the error image is the difference between the original and the reconstructed images. These values are tabulated in Table 2.

Example 2. This example is carried out for the design of a $\mathrm{HH}$ filter with cut-off frequencies $\left(\omega_{s_{1}}, \omega_{s_{2}}\right)=(0.80 \pi, 0.30 \pi)$ in order to test the performances of the NSPRL filter and the 3PLF under nonsymmetrical filter conditions. For each order, the lattice filter parameters and the stopband average power values are given in Table 3 . The subband and the reconstructed images are shown in Figures 12 and 13, respectively, and the SNR values are tabulated in Table 4. The PR property of the proposed method is observed even though the cut-off frequencies in each direction are not equal.

In Example 1, the first and the third coefficients of the lattice filter $\left(k_{1}^{(2 m+1)}\right.$ and $\left.k_{3}^{(2 m+1)}\right)$ are observed to be equal although they are not constrained to be so. This is the consequence of having square-shaped filter characteristics in the frequency domain (i.e., both of the stopband edge frequencies are selected identical). When the two stopband edge frequencies are taken to be different as in Example 2, these two lattice filter coefficients are unequal as seen in Table 3.

When the autocorrelation functions of the original and the subband images are examined, it is seen that LL subimage contains significantly more energy than the others do. This means that the proposed NSPRL filter structure has a good energy compaction performance and compacts most of the energy in the LL subband.

\section{CONCLUSIONS}

In this paper, we present a new lattice filter structure for the design of a nonseparable 2D four-band subband decomposition system. First, the analysis lattice filter structure is constructed according to the frequency specifications of the four subband filters and then the synthesis filter is obtained using the polyphase representation of the subband filter bank satisfying the requirements for alias-free and PR conditions. The PR solution, however, requires orthonormal filter design which decreases the number of independent filter parameters from three to two. The proposed 2D four-channel NSPRL filter bank reconstructs the original image exactly, in the absence of decoding/encoding of the subbands, with no restrictions on the symmetry conditions of the desired frequency characteristics and the filter length. It is also shown 
TABLE 3: Results of the filter design with $\left(\omega_{s_{1}}, \omega_{s_{2}}\right)=(0.80 \pi, 0.80 \pi)$.

\begin{tabular}{c|c|ccc|c}
\hline Filter structure & Order & \multicolumn{3}{|c|}{ Lattice filter coefficients } & Stopband average power \\
\hline \multirow{3}{*}{ NSPRL } & & $k_{1}$ & $k_{2}$ & $k_{3}$ & 0.7987 \\
& 1 & -1 & -1 & -1 & 0.0979 \\
& 3 & 0.2428 & -0.0238 & 0.0319 & 0.7823 \\
& 5 & -0.0831 & 0.0027 & -0.0175 & 0.7821 \\
\hline \multirow{3}{*}{ 3PLF } & 7 & 0.0296 & 0.0005 & -0.1132 & 0.9638 \\
& 1 & -0.0593 & -0.0554 & -0.0639 & 0.9557 \\
& 3 & 0.0027 & 0.0019 & -0.0126 & 0.9554 \\
& 5 & 0.0063 & -0.0008 & 0.0050 & 0.9553 \\
\hline
\end{tabular}
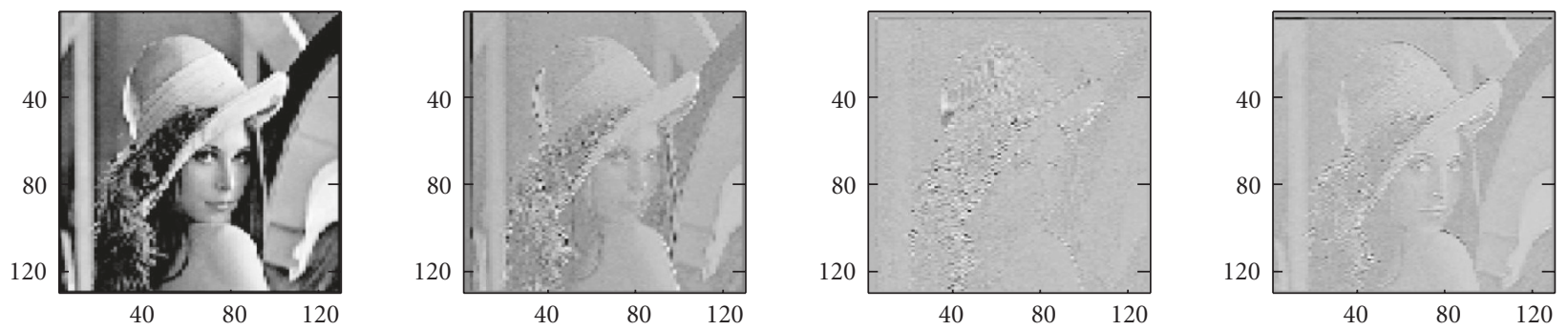

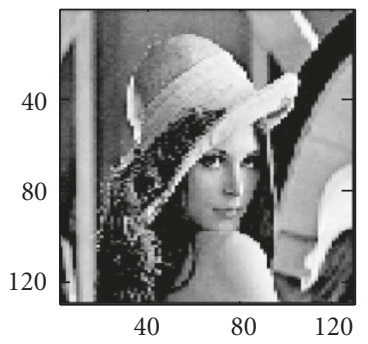

(a)

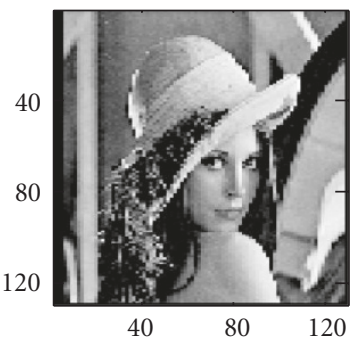

(b)

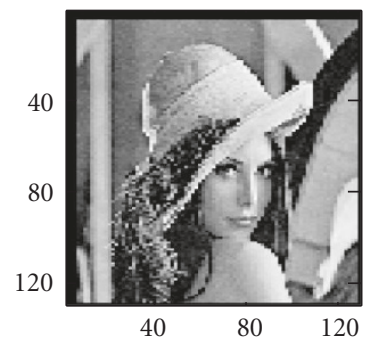

(c)

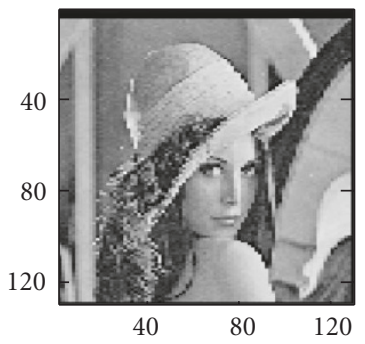

(d)

FIgURE 12: The subimages obtained using (1st row) the 7th-order NSPRL subband filters; (2nd row) 7th-order 3PLF subband filters-all $\left(\omega_{s_{1}}, \omega_{s_{2}}\right)=(0.80 \pi, 0.30 \pi)$. (a) LL subimages; (b) HL subimages; (c) HH subimages; (d) LH subimages.

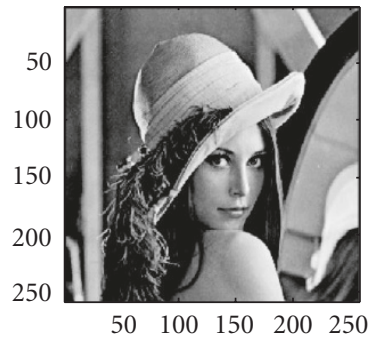

(a)

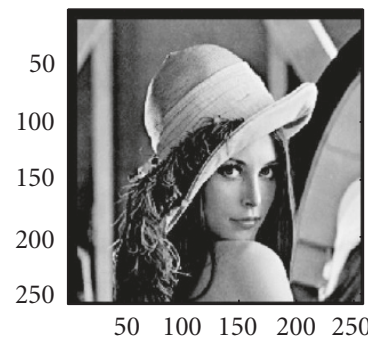

(b)

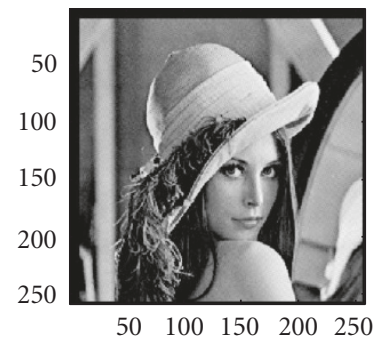

(c)

FIGURE 13: (a) The original image; (b) the reconstructed image using the proposed NSPRL filter bank; (c) the reconstructed image using the 3 PLF—all for $\left(\omega_{s_{1}}, \omega_{s_{2}}\right)=(0.80 \pi, 0.30 \pi)$.

that the SPRL filter bank constructed from the 1D lattice filter using the design parameters of [14] is a special case of the proposed NSPRL filter bank under four-quadrant symmetry conditions.
The proposed structure is easy to implement and can be used in image compression where each subband can be encoded using appropriate techniques. Moreover, this structure not only allows the design of square-shaped filters, but 
TABLE 4: SNR values related to reconstruction by different filter banks for $\left(\omega_{s_{1}}, \omega_{s_{2}}\right)=(0.80 \pi, 0.30 \pi)$.

\begin{tabular}{cc}
\hline Filter structure & SNR $(\mathrm{dB})$ \\
\hline NSPRL & 81.11 \\
3PLF & 18.68 \\
\hline
\end{tabular}

also that of rectangular-shaped filters. This is in fact a consequence of the nonseparable approach and thus, the proposed filter can be used for processing special 2D data to concentrate signal energy into fewer subbands.

\section{REFERENCES}

[1] R. E. Crochiere, S. A. Webber, and J. L. Flanagan, "Digital coding of speech in subbands," Bell System Technical Journal, vol. 55, no. 8, pp. 1069-1085, 1976.

[2] M. Vetterli, "Multidimensional subband coding: some theory and algorithms," Signal Processing, vol. 6, no. 2, pp. 97-112, 1984.

[3] J. W. Woods and S. D. O'neil, "Subband coding of images," IEEE Transactions on Acoustics, Speech, and Signal Processing, vol. 34, no. 5, pp. 1278-1288, 1986.

[4] M. J. T. Smith and S. L. Eddins, "Analysis/synthesis techniques for subband image coding," IEEE Transactions on Acoustics, Speech, and Signal Processing, vol. 38, no. 8, pp. 1446-1456, 1990.

[5] I. Daubechies, "Orthonormal bases of compactly supported wavelets," Communications on Pure and Applied Mathematics, vol. 41, pp. 909-996, 1988.

[6] S. G. Mallat, "Multifrequency channel decompositions of images and wavelet models," IEEE Transactions on Acoustics, Speech, and Signal Processing, vol. 37, no. 12, pp. 2091-2110, 1989.

[7] C. R. Galand and H. J. Nussbaumer, "New quadrature mirror filter structures," IEEE Transactions on Acoustics, Speech, and Signal Processing, vol. 32, no. 3, pp. 522-531, 1984.

[8] V. K. Jain and R. E. Crochiere, "Quadrature mirror filter design in the time domain," IEEE Transactions on Acoustics, Speech, and Signal Processing, vol. 32, no. 2, pp. 353-361, 1984.

[9] M. J. T. Smith and T. P. Barnwell, "Exact reconstruction techniques for tree-structured subband coders," IEEE Transactions on Acoustics, Speech, and Signal Processing, vol. 34, no. 3, pp. 434-441, 1986.

[10] P. P. Vaidyanathan, "Quadrature Mirror Filter banks, $M$-Band extensions, and PR techniques," IEEE Acoustics, Speech, and Signal Processing Magazine, vol. 4, no. 3, pp. 4-20, 1987.

[11] P. P. Vaidyanathan, "Theory and design of $M$-channel maximally decimated quadrature mirror filters with arbitrary $M$, having the perfect reconstruction property," IEEE Transactions on Acoustics, Speech, and Signal Processing, vol. 35, no. 4, pp. 476-492, 1987.

[12] M. Vetterli and D. J. Le Gall, "Perfect reconstruction FIR filter banks: some properties and factorizations," IEEE Transactions on Acoustics, Speech, and Signal Processing, vol. 37, no. 7, pp. 1057-1071, 1989.

[13] T. Q. Nguyen and P. P. Vaidyanathan, "Two-channel perfect reconstruction FIR QMF structures which yield linear phase analysis and synthesis filters," IEEE Transactions on Acoustics, Speech, and Signal Processing, vol. 38, no. 3, pp. 433-446, 1990.

[14] P. P. Vaidyanathan and P. Q. Hoang, "Lattice structures for optimal design and robust implementation of two-channel perfect-reconstruction QMF banks," IEEE Transactions on Acoustics, Speech, and Signal Processing, vol. 36, no. 1, pp. 8194, 1988.

[15] A. N. Akansu and R. A. Haddad, Multiresolution Signal Decomposition: Transforms, Subbands and Wavelets, Academic Press, San Diego, Calif, USA, 1992.

[16] D. B. H. Tay and N. G. Kingsbury, "Flexible design of multidimensional perfect reconstruction FIR 2-band filters using transformations of variables," IEEE Transactions on Image Processing, vol. 2, no. 4, pp. 466-480, 1993.

[17] E. P. Simoncelli and E. H. Adelson, "Non-separable extensions of quadrature mirror filters to multiple dimensions," Proceedings of the IEEE, vol. 78, no. 4, pp. 652-664, 1990, Special issue on multi-dimensional signal processing.

[18] R. H. Bamberger and M. J. T. Smith, "A filter bank for the directional decomposition of images: theory and design," IEEE Transactions on Signal Processing, vol. 40, no. 4, pp. 882-893, 1992.

[19] R. Ansari and C. L. Lau, "Two dimensional IIR filters for exact reconstruction in tree-structured sub-band decomposition," Electronics Letters, vol. 23, no. 12, pp. 633-634, 1987.

[20] R. Ansari, "Efficient IIR and FIR fan filters," IEEE Transactions on Circuits and Systems, vol. 34, no. 8, pp. 941-945, 1987.

[21] T. Chen and P. P. Vaidyanathan, "Multidimensional multirate filters derived from one dimensional filters," Electronics Letters, vol. 27, no. 3, pp. 225-228, 1991.

[22] T. Chen and P. P. Vaidyanathan, "Multidimensional multirate filters and filter banks derived from one-dimensional filters," IEEE Transactions on Signal Processing, vol. 41, no. 5, pp. 17491765, 1993.

[23] E. Viscito and J. P. Allebach, "Design of perfect reconstruction multi-dimensional filter banks using cascaded Smith form matrices," in Proceedings of IEEE International Symposium on Circuits and Systems (ISCAS '88), vol. 1, pp. 831-834, Espoo, Finland, June 1988.

[24] E. Viscito and J. P. Allebach, "The analysis and design of multidimensional FIR perfect reconstruction filter banks for arbitrary sampling lattices," IEEE Transactions on Circuits and Systems, vol. 38, no. 1, pp. 29-41, 1991.

[25] S. Basu and C. H. Chiang, "A complete parametrization of 2D nonseparable orthogonal wavelets," in Proceedings of IEEE International Symposium on Time-Frequency and Time-Scale Analysis, pp. 55-58, Victoria, BC, Canada, October 1992.

[26] J. Kovacevic and M. Vetterli, "Nonseparable multidimensional perfect reconstruction filter banks and wavelet bases for $\mathfrak{R}^{n}$," IEEE Transactions on Information Theory, vol. 38, no. 2, pp. 533-555, 1992, Special issue on wavelet transform and multiresolution signal analysis.

[27] J. Kovaceciv and M. Vetterli, "Perfect reconstruction filter banks with rational sampling factors," IEEE Transactions on Signal Processing, vol. 41, no. 6, pp. 2047-2066, 1993.

[28] S. Venkataraman and B. C. Levy, "Nonseparable orthogonal linear phase perfect reconstruction filter banks and their application to image compression," in Proceedings of the International Conference on Image Processing (ICIP '94), pp. 334-338, Austin, Tex, USA, November 1994.

[29] X. Q. Gao, T. Q. Nguyen, and G. Strang, "Theory and lattice structures of complex paraunitary filterbanks with filters of (Hermitian)-symmetry/antisymmetry properties," IEEE Transactions on Signal Processing, vol. 49, no. 5, pp. 10281043, 2001.

[30] S. C. Chan, "Two-dimensional nonseparable modulated filter banks," in Proceedings of the IEEE International Conference on 
Acoustics, Speech, and Signal Processing (ICASSP '94), pp. 141144, Adelaide, Australia, April 1994.

[31] J. Kovacevic and M. Vetterli, "Nonseparable two- and threedimensional wavelets," IEEE Transactions on Signal Processing, vol. 43, no. 5, pp. 1269-1273, 1995.

[32] T. D. Tran and T. Q. Nguyen, "On $M$-Channel linear phase FIR Filter banks and application in image compression," IEEE Transactions on Signal Processing, vol. 45, no. 9, pp. 2175-2187, 1997.

[33] J. Huang and G. Gu, "A direct approach to the design of QMF banks via frequency domain optimization," IEEE Transactions on Signal Processing, vol. 46, no. 8, pp. 2131-2146, 1998.

[34] G. Evangelista and S. Cavaliere, "Frequency warped filter banks and wavelet transform: a discrete-time approach via laguerre expansions," IEEE Transactions on Signal Processing, vol. 46, no. 10, pp. 2638-2650, 1998.

[35] C. K. Goh and Y. C. Lim, "An efficient algorithm to design weighted minimax perfect reconstruction quadrature mirror filter banks," IEEE Transactions on Signal Processing, vol. 47, no. 12, pp. 3303-3314, 1999.

[36] F. Labeau, L. Vandendorpe, and B. Macq, "Structures, factorizations and design criteria for oversampled paraunitary filterbanks yielding linear-phase filters," IEEE Transactions on Signal Processing, vol. 48, no. 11, pp. 3062-3071, 2000.

[37] T. D. Tran, M. Ikehara, and T. Q. Nguyen, "Linear phase paraunitary filter bank with filters of different lengths and its application in image compression," IEEE Transactions on Signal Processing, vol. 47, no. 10, pp. 2730-2744, 1999.

[38] X. Gao, Q. Nguyen, and G. Strang, "On factorization of $M$ channel paraunitary filterbanks," IEEE Transactions on Signal Processing, vol. 49, no. 5, pp. 1433-1446, 2001.

[39] S. Oraintara, T. D. Tran, P. N. Heller, and T. Q. Nguyen, "Lattice structure for regular paraunitary linear-phase filterbanks and $M$-band orthogonal symmetric wavelets," IEEE Transactions on Signal Processing, vol. 49, no. 11, pp. 2659-2672, 2001.

[40] S. R. Parker and A. H. Kayran, "Lattice parameter autoregressive modelling of 2-D fields-Part I: the quarter plane case," IEEE Transactions on Acoustics, Speech, and Signal Processing, vol. 32, no. 4, pp. 872-885, 1984.

[41] H. Lev-Ari and S. R. Parker, "Stable and efficient 2-D lattice filters," in Proceedings of the IEEE International Conference on Acoustics, Speech, and Signal Processing (ICASSP '86), pp. 695698, Tokyo, Japan, April 1986.

[42] S. Sezen, "Application of 2-D lattice filter structures to the subband decomposition problem," M.S. thesis, Boğaziçi University, Istanbul, Turkey, 1998.

[43] A. Ertuzun, A. H. Kayran, and E. Panayirci, "Stable quarterplane 2-D lattice filters," Electronic Letters, vol. 26, no. 12, pp. 806-807, 1990.

S. Sezen was born in 1972 in Istanbul, Turkey. He received his Bachelor's degree and Master of Science degree in electrical and electronic engineering from Boğaziçi University, Istanbul, Turkey in 1995 and 1998, respectively. He has conducted researches in the areas of adaptive filter theory, lattice filter structures, quadrature mirror filter banks, utilization of lattice filters in 2D subband decomposition and perfect re-

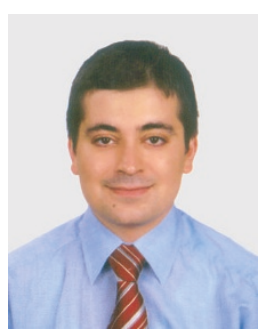
construction areas. He contributed to the preparation of 5 scientific conference papers on lattice filter structures and their applications.
Since 1996, he has been working for Siemens AG, Istanbul, in process automation engineering, test and commissioning, project and proposals management for cement business.

A. Ertüzün was born in 1959 in Salihli, Turkey. She received the B.S. degree (with honors) from Boğaziçi University, Istanbul, Turkey, the M. Eng. degree from McMaster University, Hamilton, Ontario, Canada, and the Ph.D. degree from Boğaziçi University, Istanbul, Turkey, all in electrical engineering, in 1981, in 1984, and in 1989, respectively. Since 1988, she has been with the Department of Electrical and Electronic En-

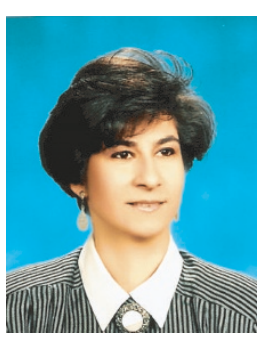
gineering at Boğaziçi University where she is currently an Associate Professor. Her current research interests are in the areas of independent component analysis and its applications, blind signal processing, Bayesian methods, adaptive systems with applications to communication systems, image processing, and texture analysis. She has authored and coauthored more than 60 scientific papers in journals and conference proceedings. She is a Member of IEEE Signal Processing and Communication Societies and IAPR (International Association of Pattern recognition) and IEICE (The Institute of Electronics Information and Communication Engineers). 